\title{
Enhanced Micromechanical Modelling of Martensitic Phase-Transitions Considering Plastic Deformations
}

\author{
Thorsten Bartel ${ }^{1, a}$, Andreas Menzel ${ }^{12}$, and Bob Svendsen ${ }^{1}$ \\ 1 TU Dortmund, Institute of Mechanics, Dortmund, GER \\ 2 Lund University, Division of Solid Mechanics, Lund, SWE
}

\begin{abstract}
The purpose of this contribution is the presentation of a micromechanical model for martensitic phase transformations which can be applied to a wide range of materials like shape memory alloys (SMA), TRIP-steels (TRansformation Induced Plasticity) and piezoceramics. One of the key-features of the model is the consideration of several martensitic variants in addition to the parent phase austenite based on crystallographic theories. According to a specifically chosen microstructure, a fluctuation field is superimposed to the local, homogeneous deformations. These fluctuations implicate several additional internal variables which are partially supposed to minimize the microscopic energy density. Furthermore, the variables assumed to be 'dissipative', like the volume fractions of martensite, are determined by evolution laws. Another focal point of our work is the combination of martensitic phase transformations and plasticity. As a first step towards a complete micromechanical description of this problem, we make use of a phenomenological approach for plasticity here. The results of our computations reveal significant differences of the single-crystalline behavior to the well-known macroscopic material response, which is indeed verified by experimental studies.
\end{abstract}

\section{Introduction}

Recent developments in the field of materials science, especially concerning so-called "smart materials" like SMAs, TRIP-steels and piezoceramics, require a sound mechanical modelling of such materials. In this context, our aim is to follow theories as for example presented in [1-6]. The concepts provided therein are closely related to the so-called quasi-convexification introduced in [7] and the direct methods in the calculus of variations (see for example $[8,9]$ ). Here, we adopt the upper energy bound presented in $[10,11]$ for the part of solid-solid phase transformations. Similar approaches are stated for example in [12-14]. A significant interaction between martensitic transformations and plasticity for SMAs has been observed for example by $[15-17]$. The combination of pseudoelasticity with plasticity in the framework of micromechanical models is still rather rare in literature. Here, we use an additive decomposition of total strains into elastic, transformation and plastic strains within each phase. This leads to the coupling of both fields: the phase microstructure and the "smeared-out" dislocation microstructure represented by the phenomenological plastic strain tensors. Similar approaches can be found in $[18,19]$. For simplicity, we follow [20] and work with a classical von Mises-type plasticity formulation here.

\footnotetext{
a e-mail: thorsten. bartel@udo.edu
} 


\section{Kinematics}

Since the model formulation is restricted to small deformations, the additive decomposition of strains

$$
\varepsilon=\varepsilon^{\mathrm{el}}+\varepsilon^{\mathrm{tr}}+\varepsilon^{\mathrm{pl}}
$$

is used. Here, $\varepsilon^{\text {tr }}$ can be deduced from the change of the crystal structure during a phase change according to [21]. Correspondingly, these are also known as Bain strains and can be chosen as stated in [22], for instance, depending on the crystal structure of martensite. This results in $N V$

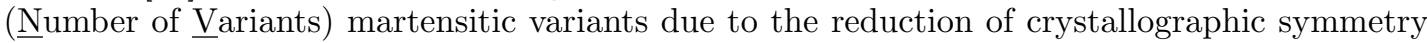
along with $\overline{N V}$ different transformation strains $\varepsilon_{I}^{\mathrm{tr}}$. In contrast to these material parameters, $\varepsilon^{\mathrm{pl}}$ reflects phenomenological, tensor-valued internal variables for the plastic deformations. The multiphase formulation of the model requires the introduction of such variables for each phase, namely $\varepsilon_{A}^{\mathrm{pl}}$ (austenite) and $\varepsilon_{I}^{\mathrm{pl}}, I=1 \ldots N V$ ( $I$-th martensitic variant). Moreover, it is well known that a significant reduction of the energy can be achieved by assuming that different total strains are present within each phase of the material. This leads to the following decomposition of strains:

$$
\varepsilon= \begin{cases}\varepsilon_{A}=\varepsilon_{A}^{\mathrm{el}}+\varepsilon_{A}^{\mathrm{pl}} & \text { for austenite } \\ \varepsilon_{I}=\varepsilon_{I}^{\mathrm{el}}+\varepsilon_{I}^{\mathrm{tr}}+\varepsilon_{I}^{\mathrm{pl}} & \text { for the } I \text {-th martensitic variant }\end{cases}
$$

\section{Constitutive model}

Below, a suitable energy density for the phase-mixture is derived. The first step is to define energy densities for each phase of the material. Here, we choose quadratic forms

$$
\begin{aligned}
\psi_{A}\left(\varepsilon_{A}^{\mathrm{el}}\right):=\frac{1}{2}\left(\varepsilon_{A}-\varepsilon_{A}^{\mathrm{pl}}\right): C_{A}:\left(\varepsilon_{A}-\varepsilon_{A}^{\mathrm{pl}}\right)+c_{A} \\
\psi_{I}\left(\varepsilon_{I}^{\mathrm{el}}\right):=\frac{1}{2}\left(\varepsilon_{I}-\varepsilon_{I}^{\mathrm{tr}}-\varepsilon_{I}^{\mathrm{pl}}\right): C_{M}:\left(\varepsilon_{I}-\varepsilon_{I}^{\mathrm{tr}}-\varepsilon_{I}^{\mathrm{pl}}\right)+c_{M}
\end{aligned}
$$

where $\boldsymbol{C}_{A}, \boldsymbol{C}_{M}$ denote elasticity tensors for austenite and martensite, respectively, and $c_{A}$, $c_{M}$ are referred to as chemical energies, which take into account temperature dependencies. Together with the volume fractions of martensite, $\theta_{I}$, the averaged energy density for the phase mixture

$$
\bar{\psi}=\left(1-\sum_{I=1}^{N V} \theta_{I}\right) \psi_{A}\left(\varepsilon_{A}^{\mathrm{el}}\right)+\sum_{I=1}^{N V} \theta_{I} \psi_{I}\left(\varepsilon_{I}^{\mathrm{el}}\right)
$$

is defined. Of course, the total strains of each phase $\varepsilon_{A}$ and $\varepsilon_{M}$ are not independent of the macroscopic strains $\varepsilon$. Here, these quantities are derived from a fluctuation field $\varphi$ superimposed to the local, homogeneous deformations. The specific ansatz for the fluctuation field is taken from $[10,11]$. There, a laminated microstructure is applied dividing a representative volume element into regions of austenite $\left(\Omega_{A}\right)$ and patterns for all martensitic variants $\left(\Omega_{I}\right)$ as depicted in Fig. 1. According to this, the $C_{0}$-smooth vectorial fluctuation field is defined as

$$
\boldsymbol{\varphi}(\boldsymbol{x})= \begin{cases}\frac{1}{1-\theta_{M}} \boldsymbol{u}_{A}(\xi-\mathcal{K}+1) & , \text { if } \boldsymbol{x} \in \Omega_{A} \\ -\frac{1}{\theta_{M}} \boldsymbol{u}_{A}(\xi-\mathcal{K}) & , \text { if } \boldsymbol{x} \in \Omega_{I} \\ +\frac{1}{\theta_{I}}\left(\boldsymbol{u}_{I}-\boldsymbol{u}_{I-1}\right)\left(\zeta-\sum_{k=1}^{I-1}\left\{\theta_{k}\right\}-(\mathcal{M}-1) \theta_{M}\right) & \\ +\boldsymbol{u}_{I-1} & \end{cases}
$$

where $\theta_{M}:=\sum_{I=1}^{N V} \theta_{I}, \xi:=\boldsymbol{x} \cdot \boldsymbol{n}_{A}, \zeta:=\boldsymbol{x} \cdot \boldsymbol{n}_{M}$ and $\mathcal{K}, \mathcal{M}$ denote the current number of the considered pattern of the periodic field along the orientation vectors of the austenite and martensite laminates $\boldsymbol{n}_{A}$ and $\boldsymbol{n}_{M}$, respectively. Furthermore, $\boldsymbol{u}_{A}, \boldsymbol{u}_{I}$ symbolize the so-called amplitudes 


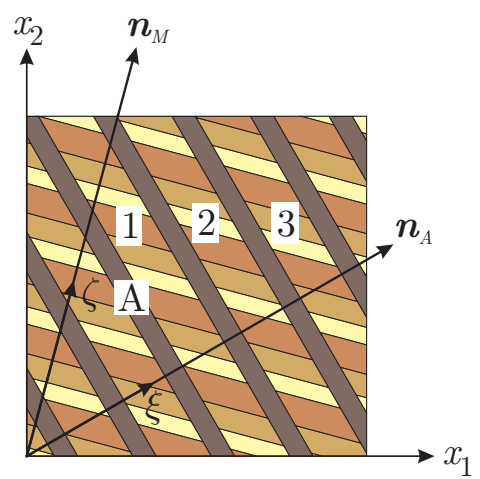

Fig. 1. Applied microstructure to the material's microscale after $[10,11]$ (for $N V=3$ ).

of $\boldsymbol{\varphi}$. For further details, especially in terms of a graphical illustration of the fluctuation field, we once again refer to [11]. With this at hand, one obtains

$$
\begin{aligned}
\boldsymbol{\varepsilon}_{A} & =\boldsymbol{\varepsilon}+\frac{1}{1-\theta_{M}} \boldsymbol{n}_{A} \otimes_{\mathrm{sym}} \boldsymbol{u}_{A} \\
\boldsymbol{\varepsilon}_{I} & =\boldsymbol{\varepsilon}-\frac{1}{\theta_{M}} \boldsymbol{n}_{A} \otimes_{\mathrm{sym}} \boldsymbol{u}_{A}+\frac{1}{\theta_{I}} \boldsymbol{n}_{M} \otimes_{\mathrm{sym}}\left(\boldsymbol{u}_{I}-\boldsymbol{u}_{I-1}\right)
\end{aligned}
$$

with $\boldsymbol{a} \otimes_{\text {sym }} \boldsymbol{b}:=\frac{1}{2}(\boldsymbol{a} \otimes \boldsymbol{b}+\boldsymbol{b} \otimes \boldsymbol{a}), \boldsymbol{u}_{0}=\boldsymbol{u}_{N V}=\mathbf{0}$ in a straight forward way via $\boldsymbol{\varepsilon}=$ $1 / 2(\nabla(\boldsymbol{u}+\boldsymbol{\varphi})+(\boldsymbol{u}+\boldsymbol{\varphi}) \nabla)$. This ansatz fulfills compatibility of strains in the sense that $\left(1-\theta_{M}\right) \varepsilon_{A}+\sum_{I} \theta_{I} \varepsilon_{I}=\varepsilon$ holds and a symmetrized rank-one connection between the phase strains is achieved in the spirit of $[23,22,24]$. In the following, the evolution of $\boldsymbol{u}_{A}, \boldsymbol{u}_{I}$ is assumed to be purely energetic in nature. Hence, they are determined via the stationarity conditions

$$
\begin{aligned}
\frac{\partial \bar{\psi}}{\partial \boldsymbol{u}_{A}} & =\mathbf{0}, \\
\frac{\partial \bar{\psi}}{\partial \boldsymbol{u}_{I}} & =\mathbf{0} \quad, I=1, \ldots, N V-1 .
\end{aligned}
$$

The solution of these yields

$$
\begin{aligned}
\boldsymbol{u}_{A} & =-\widehat{\boldsymbol{C}}^{-1} \cdot\left(\Delta \boldsymbol{C}: \boldsymbol{\varepsilon}+\frac{1}{\theta_{M}}\left(\overline{\boldsymbol{\tau}}^{\mathrm{tr}}+\overline{\boldsymbol{\tau}}_{M}^{\mathrm{pl}}\right)-\boldsymbol{\tau}_{A}^{\mathrm{pl}}\right) \cdot \boldsymbol{n}_{A} \\
\boldsymbol{u}_{I} & =-\frac{1}{\theta_{M}} \widehat{\boldsymbol{C}}_{M}^{-1} \cdot\left(\sum_{i=1}^{I} \sum_{j=I+1}^{N V} \theta_{i} \theta_{j}\left(\boldsymbol{\tau}_{j}^{\mathrm{tr}}+\boldsymbol{\tau}_{j}^{\mathrm{pl}}-\boldsymbol{\tau}_{i}^{\mathrm{tr}}-\boldsymbol{\tau}_{i}^{\mathrm{pl}}\right)\right) \cdot \boldsymbol{n}_{M}
\end{aligned}
$$

Here and further on, the following abbreviations have been used: $\overline{\boldsymbol{C}}:=\theta_{A} \boldsymbol{C}_{A}+\theta_{M} \boldsymbol{C}_{M}$, $\Delta \boldsymbol{C}:=\boldsymbol{C}_{A}-\boldsymbol{C}_{M}, \tilde{\boldsymbol{C}}:=\frac{1}{\theta_{A}} \boldsymbol{C}_{A}+\frac{1}{\theta_{M}} \boldsymbol{C}_{M}, \widehat{\boldsymbol{C}}:=\boldsymbol{n}_{A} \cdot \tilde{\boldsymbol{C}} \cdot \boldsymbol{n}_{A}, \widehat{\boldsymbol{C}}_{M}:=\boldsymbol{n}_{M} \cdot \boldsymbol{C}_{M} \cdot \boldsymbol{n}_{M}$, $\boldsymbol{\tau}_{A}^{\mathrm{pl}}:=C_{A}: \varepsilon_{A}^{\mathrm{pl}}, \boldsymbol{\tau}_{I}^{\mathrm{tr}}:=C_{M}: \varepsilon_{I}^{\mathrm{tr}}, \boldsymbol{\tau}_{I}^{\mathrm{pl}}:=C_{M}: \varepsilon_{I}^{\mathrm{pl}}, \overline{\boldsymbol{\tau}}_{A}^{\mathrm{pl}}:=\theta_{A} \boldsymbol{\tau}_{A}^{\mathrm{pl}}, \overline{\boldsymbol{\tau}}_{M}^{\mathrm{pl}}:=\sum_{i=1}^{N V} \theta_{I} \boldsymbol{\tau}_{I}^{\mathrm{pl}}$, $\overline{\boldsymbol{\tau}}^{\mathrm{tr}}:=\sum_{i=1}^{N V} \theta_{I} \boldsymbol{\tau}_{I}^{\mathrm{tr}}$. With these derivations at hand the so-called relaxed energy density can be 
represented as

$$
\begin{aligned}
\psi_{\mathrm{rel}}= & \frac{1}{2} \boldsymbol{\varepsilon}: \overline{\boldsymbol{C}}: \boldsymbol{\varepsilon}-\left(\overline{\boldsymbol{\tau}}_{M}^{\mathrm{pl}}+\overline{\boldsymbol{\tau}}_{A}^{\mathrm{pl}}+\overline{\boldsymbol{\tau}}^{\mathrm{tr}}\right): \boldsymbol{\varepsilon}+\frac{1}{2} \overline{\boldsymbol{\tau}}_{A}^{\mathrm{pl}}: \boldsymbol{\varepsilon}_{A}^{\mathrm{pl}} \\
& +\sum_{I=1}^{N V}\left\{\theta_{I} \boldsymbol{\tau}_{I}^{\mathrm{tr}}: \varepsilon_{I}^{\mathrm{pl}}\right\}+\sum_{I=1}^{N V}\left\{\frac{1}{2} \theta_{I}\left(\boldsymbol{\tau}_{I}^{\mathrm{tr}}: \boldsymbol{\varepsilon}_{I}^{\mathrm{tr}}+\boldsymbol{\tau}_{I}^{\mathrm{pl}}: \varepsilon_{I}^{\mathrm{pl}}\right)\right\} \\
& +\boldsymbol{\varepsilon}:\left(\left(\Delta \boldsymbol{C} \cdot \boldsymbol{n}_{A}\right) \cdot \boldsymbol{u}_{A}\right)+\frac{1}{2} \boldsymbol{u}_{A} \cdot \widehat{\boldsymbol{C}} \cdot \boldsymbol{u}_{A}+\frac{1}{\theta_{M}}\left(\overline{\boldsymbol{\tau}}^{\mathrm{tr}} \cdot \boldsymbol{n}_{A}\right) \cdot \boldsymbol{u}_{A} \\
& -\sum_{I=1}^{N V}\left\{\boldsymbol{\tau}_{I}^{\mathrm{tr}} \cdot\left(\boldsymbol{u}_{I}-\boldsymbol{u}_{I-1}\right)\right\} \cdot \boldsymbol{n}_{M}+\theta_{A} c_{A}+\theta_{M} c_{M} \\
& +\sum_{I=1}^{N V}\left\{\frac{1}{2 \theta_{I}}\left(\boldsymbol{u}_{I}-\boldsymbol{u}_{I-1}\right) \cdot \widehat{\boldsymbol{C}}_{M} \cdot\left(\boldsymbol{u}_{I}-\boldsymbol{u}_{I-1}\right)\right\} \\
& +\left(\left(\frac{1}{\theta_{M}} \overline{\boldsymbol{\tau}}_{M}^{\mathrm{pl}}-\boldsymbol{\tau}_{A}^{\mathrm{pl}}\right) \cdot \boldsymbol{n}_{A}\right) \cdot \boldsymbol{u}_{A}-\sum_{I=1}^{N V}\left\{\left(\boldsymbol{\tau}_{I}^{\mathrm{pl}} \cdot\left(\boldsymbol{u}_{I}-\boldsymbol{u}_{I-1}\right)\right) \cdot \boldsymbol{n}_{M}\right\}
\end{aligned}
$$

The effective material response in terms of elastic stresses is given by

$$
\begin{aligned}
\boldsymbol{\sigma}:= & \frac{\partial \psi_{r e l}}{\partial \boldsymbol{\varepsilon}} \\
= & \overline{\boldsymbol{C}}: \boldsymbol{\varepsilon}-\left(\overline{\boldsymbol{\tau}}_{M}^{\mathrm{pl}}+\overline{\boldsymbol{\tau}}_{A}^{\mathrm{pl}}+\overline{\boldsymbol{\tau}}^{\mathrm{tr}}\right)-\boldsymbol{\varepsilon}:\left(\left(\Delta \boldsymbol{C} \cdot \boldsymbol{n}_{A}\right) \cdot \widehat{\boldsymbol{C}}^{-1} \cdot\left(\boldsymbol{n}_{A} \cdot \Delta \boldsymbol{C}\right)\right) \\
& -\frac{1}{\theta_{M}}\left(\Delta \boldsymbol{C} \cdot \boldsymbol{n}_{A}\right) \cdot \widehat{\boldsymbol{C}}^{-1} \cdot\left(\overline{\boldsymbol{\tau}}^{\mathrm{tr}} \cdot \boldsymbol{n}_{A}\right) \\
& -\left(\left(\frac{1}{\theta_{M}} \overline{\boldsymbol{\tau}}_{M}^{\mathrm{pl}}-\boldsymbol{\tau}_{A}^{\mathrm{pl}}\right) \cdot \boldsymbol{n}_{A}\right) \cdot \widehat{\boldsymbol{C}}^{-1} \cdot\left(\boldsymbol{n}_{A} \cdot \Delta \boldsymbol{C}\right) .
\end{aligned}
$$

\section{Evolution laws}

Since the internal variables $\theta_{I}, \boldsymbol{n}_{A}, \boldsymbol{n}_{M}, \varepsilon_{A}^{\mathrm{pl}}$ and $\varepsilon_{I}^{\mathrm{pl}}$ are of dissipative character, they are treated via evolution laws. Precisely, the set of ordinary differential equations reads

$$
\begin{aligned}
\dot{\theta}_{I} & =\lambda_{I}^{\mathrm{tr}} \operatorname{sign}\left(q_{I}\right) \\
\dot{\boldsymbol{\varepsilon}}_{A}^{\mathrm{pl}} & =\lambda_{A}^{\mathrm{pl}} \tilde{\boldsymbol{q}}_{A}^{\mathrm{pl}} /\left\|\tilde{\boldsymbol{q}}_{A}^{\mathrm{pl}}\right\| \\
\dot{\boldsymbol{\varepsilon}}_{I}^{\mathrm{pl}} & =\lambda_{I}^{\mathrm{pl}} \tilde{\boldsymbol{q}}_{I}^{\mathrm{pl}} /\left\|\tilde{\boldsymbol{q}}_{I}^{\mathrm{pl}}\right\|
\end{aligned}
$$

for $I=1, \ldots, N V$ along with Kuhn-Tucker-type equations/inequalities

$$
\begin{array}{llll}
\Phi_{I}^{\mathrm{tr}} \lambda_{I}^{\mathrm{tr}}=0 & , \quad \Phi_{I}^{\mathrm{tr}} \leq 0 & , & \lambda_{I}^{\mathrm{tr}} \geq 0 \\
\Phi_{A}^{\mathrm{pl}} \lambda_{A}^{\mathrm{pl}}=0 & , \quad \Phi_{A}^{\mathrm{pl}} \leq 0 & , & \lambda_{A}^{\mathrm{pl}} \geq 0 \\
\Phi_{I}^{\mathrm{pl}} \lambda_{I}^{\mathrm{pl}}=0 & , \quad \Phi_{I}^{\mathrm{pl}} \leq 0 & , & \lambda_{I}^{\mathrm{pl}} \geq 0
\end{array}
$$


and yield functions

$$
\begin{aligned}
\Phi_{I}^{\mathrm{tr}} & =\left|q_{I}\right|-k\left(\alpha_{\mathrm{pl}}\right) \\
\Phi_{A}^{\mathrm{pl}} & =\left\|\tilde{\boldsymbol{q}}_{A}^{\mathrm{pl}}\right\|-\Psi_{A}\left(\alpha_{\mathrm{pl}}\right) \\
\Phi_{I}^{\mathrm{pl}} & =\left\|\tilde{\boldsymbol{q}}_{I}^{\mathrm{pl}}\right\|-\Psi_{M}\left(\alpha_{\mathrm{pl}}\right) .
\end{aligned}
$$

In (15) and (17), $\|\boldsymbol{q}\|:=\sqrt{\boldsymbol{q}: \boldsymbol{q}}$ denotes the tensorial norm and $\tilde{\boldsymbol{q}}:=\boldsymbol{q}-\frac{1}{3} \operatorname{tr}(\boldsymbol{q}) \boldsymbol{I}$ reflects the deviatoric part of $\boldsymbol{q}$. Here, we assume that the reorientation of the microstructure would require a large scale redistribution of phases and hence results in high amounts of dissipation. Thus, the spatial orientation of austenite and martensite is kept constant and correspondingly, no evolution laws need to be applied for $\boldsymbol{n}_{A}$ and $\boldsymbol{n}_{M}$. The quantities $\boldsymbol{q}$ are referred to as driving forces and are determined via

$$
q_{I}:=-\frac{\partial \psi_{r e l}}{\partial \theta_{I}}, \quad \boldsymbol{q}_{A}^{\mathrm{pl}}:=-\frac{\partial \psi_{r e l}}{\partial \varepsilon_{A}^{\mathrm{pl}}}, \quad \boldsymbol{q}_{I}^{p l}:=-\frac{\partial \psi_{r e l}}{\partial \varepsilon_{I}^{\mathrm{pl}}}
$$

In (17), $k\left(\alpha_{\mathrm{pl}}\right), \Psi_{A}\left(\alpha_{\mathrm{pl}}\right)$ and $\Psi_{M}\left(\alpha_{\mathrm{pl}}\right)$ denote threshold values for the initiation of phase transformations and plastic deformations, respectively. They depend on the equivalent plastic strain $\alpha_{\mathrm{pl}}$ which evolves according to the total amount of plastic deformations. Precisely, the isotropic hardening law

$$
\begin{aligned}
k\left(\alpha_{\mathrm{pl}}\right) & :=k_{0}+\left(k_{\infty}-k_{0}\right)\left(1-e^{-\beta_{k} \alpha_{\mathrm{pl}}}\right) \\
\Psi_{A}\left(\alpha_{\mathrm{pl}}\right) & :=\Psi_{A, 0}+\left(\Psi_{A, \infty}-\Psi_{A, 0}\right)\left(1-e^{-\beta_{A} \alpha_{\mathrm{pl}}}\right) \\
\Psi_{M}\left(\alpha_{\mathrm{pl}}\right) & :=\Psi_{M, 0}+\left(\Psi_{M, \infty}-\Psi_{M, 0}\right)\left(1-e^{-\beta_{M} \alpha_{\mathrm{pl}}}\right)
\end{aligned}
$$

is applied here. The index 0 symbolizes initial values of yield limits, whereas the index $\infty$ denotes the saturation values. The quantities $\beta$ • are additional hardening parameters.

Since the underlying system of differential-algebraic equations is knwon to be "stiff", an Aand L-stable numerical integration scheme is required in order to solve (15). Hence, the timediscretization is accomplished by a classical Backward-Euler scheme and a Newton-Raphson method is used to iteratively compute the actual values of the internal variables. A supporting mathematical and numerical analysis of quasistatic rate-independent processes is for example provided in [25].

\section{Numerical examples}

In this section we present numerical results in terms of stress-strain relations and evolution of martensitic phase fractions for a single crystalline body subjected to uniaxial tension (cycles in tension and compression) under plane stress. The underlying parameters have been chosen as $C_{11}^{A}=C_{22}^{A}=C_{33}^{A}=122.98 \mathrm{GPa}, C_{12}^{A}=C_{13}^{A}=C_{23}^{A}=60.57 \mathrm{GPa}, C_{44}^{A}=C_{55}^{A}=C_{66}^{A}=$ $31.20 \mathrm{GPa}$ for the elasticities of austenite (represented in Voigt-notation here), $C_{11}^{M}=C_{22}^{M}=$ $C_{33}^{M}=50.38 \mathrm{GPa}, C_{12}^{M}=C_{13}^{M}=C_{23}^{M}=24.81 \mathrm{GPa}, C_{44}^{M}=C_{55}^{M}=C_{66}^{M}=12.78 \mathrm{GPa}$ for martensite as well as transformation strains $\varepsilon_{1}^{\text {tr }}=\alpha \boldsymbol{e}_{1} \otimes \boldsymbol{e}_{1}+\beta \boldsymbol{e}_{2} \otimes \boldsymbol{e}_{2}+\beta \boldsymbol{e}_{3} \otimes \boldsymbol{e}_{3}, \boldsymbol{\varepsilon}_{2}^{t r}=$ $\beta \boldsymbol{e}_{1} \otimes \boldsymbol{e}_{1}+\alpha \boldsymbol{e}_{2} \otimes \boldsymbol{e}_{2}+\beta \boldsymbol{e}_{3} \otimes \boldsymbol{e}_{3}, \boldsymbol{\varepsilon}_{3}^{t r}=\beta \boldsymbol{e}_{1} \otimes \boldsymbol{e}_{1}+\beta \boldsymbol{e}_{2} \otimes \boldsymbol{e}_{2}+\alpha \boldsymbol{e}_{3} \otimes \boldsymbol{e}_{3}$ with $\alpha=0.0221$, $\beta=-0.0111$ and $c_{A}-c_{M}=0.005 \mathrm{GN} / \mathrm{m}^{2}$. For the specific choice of $k_{0}=k_{\infty}=0.0125 \mathrm{GPa}$, $\Psi_{A, 0}=\Psi_{A, \infty}=1.0 \mathrm{GPa}, \Psi_{M, \infty}=1.5 \mathrm{GPa}$ and $\beta_{M}=25.0$, and [100] crystal orientation, Fig. 2 shows the results for different values of $\Psi_{M, 0}$. For $k_{0}=0.0125 \mathrm{GPa}, k_{\infty}=0.001 \mathrm{GPa}, \beta_{k}=750.0$, $\Psi_{A, 0}=\Psi_{A, \infty}=1.0 \mathrm{GPa}, \Psi_{M, 0}=0.1 \mathrm{GPa}, \Psi_{M, \infty}=1.75 \mathrm{GPa}$ and $\beta_{M}=250.0$, a complete load cycle in compression has been applied for different initial orientations of the cubic austenite lattice represented by Miller indices in Fig. 3.

The underlying material parameters do not exactly represent a specific commercialized material. 

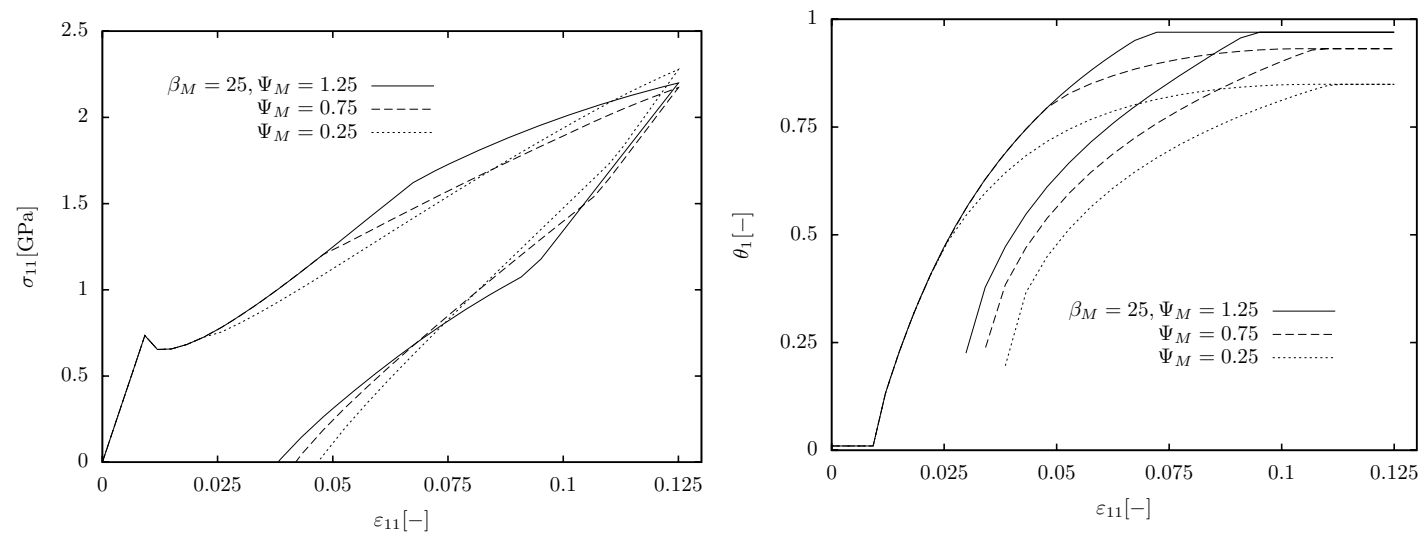

Fig. 2. stress-strain behavior and evolution of phase fraction $\theta_{1}$ depending on initiation of plastic deformations in martensite (load cycle in tension).
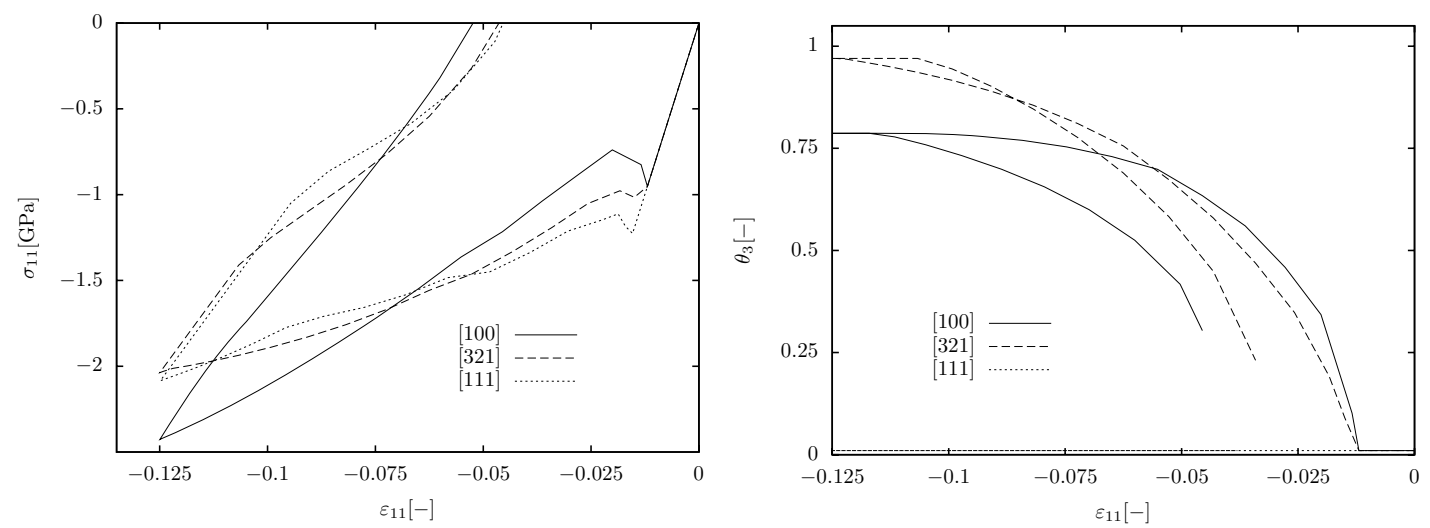

Fig. 3. stress-strain behavior and evolution of phase fraction $\theta_{3}$ depending on the initial orientation of the cubic austenite lattice (load cycle in compression).

The chosen elastic constants are based upon the assumption of isotropic behavior of each phase, which in general is neither valid for austenite nor martensite in a fully micromechanical context. The particular transformation strains, however, are taken from [22] representing typical values for the cubic to tetragonal transformation of, in this case, Indium-Thallium.

\section{Conclusion and Outlook}

In this paper, an extension of the model derived in [11] in terms of plasticity is presented. As for example verified by $[16,17]$, the material response of single crystalline aggregates may differ significantly from a macroscopic polycristalline specimen. In particular, a significant correlation between the maximum amount of martensite and the yield limit of plasticity and the initial crystal orientation of austenite is revealed, respectively.

The next step towards a fully micromechancial description is the combination with crystal plasticity. A promising future objective is the application of the presented concept to materials like TRIP-steels, piezoceramics and shape memory polymers. 


\section{References}

1. Kohn, R., Cont. Mech. Thermodyn. 3, (1991) 193-236

2. Smyshlyaev, V., Willis, J., Proc. Roy. Soc. London A 455, (1998) 779-814

3. Ortiz, M., Repetto, E., J. Mech. Phys. Sol. 47, (1999) 397-462

4. Carstensen, C., Hackl, K., Mielke, A., Proc. Roy. Soc. London A 458, (2002) 299-317

5. Govindjee, S., Mielke, A., Hall, G., J. Mech. Phys. Sol. 50, (2002) 1897-1922

6. Bartels, S., Carstensen, C., Hackl, K., Hoppe, U., Comp. Meth. Appl. Mech. Engrg. 193, (2004) 5143-5175

7. Morrey, C.B., Pac. J. Math. 2, (1952) 25-53

8. Dacorogna, B., J. Func. Anal. 46, (1982) 102-118

9. Silhavy, M., The Mechanics and Thermodynamics of Continuous Media (Springer, 1997)

10. Bartel, T., Hackl, K., Mat. Sci. Engrg. 481-482, (2008) 371-375

11. Bartel, T., Hackl, K., ZAMM accepted for publication, (2009)

12. Pagano, S., Alart, P., Maisonneuve, O., Int. J. Eng. Sci. 36, (1998) 1143-1172

13. Hackl, K., Schmidt-Baldassari, M., Mat. Sc. Eng. A 378, (2004) 503-506

14. Hackl, K., Heinen, R., Cont. Mech. Thermodyn. 19, (2008) 499-510

15. Wagner, M., Dissertation Ruhr-Universitaet Bochum, (2005)

16. Sehitoglu, H., Karaman, I., Anderson, R., Zhang, X., Gall, K., Maier, H.J., Chumlyakov, Y., Acta Mater. 48, (2000) 3311-3326

17. Hamilton, R., Sehitoglu, H., Efstathiou, C., Maier, H., Chumlyakov, Y., Zhang, X., Scripta Mater. 53, (2005) 131-136

18. Sadjadpour, A., Bhattacharya, K., Smart Mater. Struct. 16, (2007) 51-62

19. Sadjadpour, A., Bhattacharya, K., Smart Mater. Struct. 16, (2007) 1751-1765

20. Kouznetsova, V.G., Geers, M.G.D., Mech. Mat. 40, (2008) 641-657

21. Bain, E.C., Trans. AIME 70, (1924) 25

22. Bhattacharya, K., Microstructure of Martensite - Why it forms and how it gives rise to the shape memory effect (Oxford University Press, 2003)

23. Ball, J., James, R., Arch. Rat. Mech. Anal. 100, (1987) 13-52

24. James, R., Hane, K., Acta Mater. 48, (2006) 197-222

25. Mielke, A., Roubicek, T., Math. Model. Numer. Anal. 43, (2009) 399-428 